\title{
Anterior Hemi-epiphysiodesis of the Distal Tibia for Residual Equinus Deformity in Children with Clubfeet
}

\author{
Benjamin F. Giertych, $M D^{1,2}$; Sara H. Galli, $M D^{1,3}$; Matthew A. Halanski, MD ${ }^{1,4,5,6}$; Pamela J. Lang, MD ${ }^{1,4}$ \\ ${ }^{1}$ Department of Orthopedics \& Rehabilitation, University of Wisconsin, Madison, WI; ${ }^{2}$ WVU Medicine, Morgantown, WV; \\ ${ }^{3}$ OrthoCarolina, Charlotte, NC; ${ }^{4}$ American Family Children's Hospital, Madison, WI; ${ }^{5}$ University of Nebraska Medical Center, \\ Omaha, NE; ${ }^{6}$ Children's Hospital of Omaha, Omaha, NE
}

Correspondence to: Pamela J. Lang, MD; UW Medical Foundation Centennial, Building 1685 Highland Ave, 6th Floor Madison, WI 53705-2281, E-mail: PLang@ortho.wisc.edu

Received: January 1, 2022; Accepted: January 7, 2022; Published: February 1, 2022

DOI: 10.55275/JPOSNA-2022-0004

\begin{abstract}
:
Background: Residual equinus deformity in toddlers with a history of clubfoot can be managed with soft tissue release, provided there is no articular incongruity. Distal tibia osteotomy is a reasonable choice in mature patients with a flattened talus provided the distal tibia physis is closed. Anterior distal tibia hemi-epiphysiodesis is an option for children with articular incongruity who have substantial growth remaining. The aim of this study is to evaluate radiographic and clinical outcomes of this method.
\end{abstract}

Methods: Fourteen clubfoot patients (20 ankles) with residual equinus deformity were treated between 2010-2015 with anterior distal tibial hemi-epiphysiodesis utilizing size appropriate modular plate and screw constructs. Patients were evaluated clinically and radiographically. Oxford Ankle Foot Questionnaire for Children (and caregivers) with supplemental questions were requested at most recent follow-up.

Results: There were 12 males and 2 females, mean age 10 years at surgery (50-152 months), with minimum 18 months of radiographic follow-up [Mean follow-up of 34 months (Range: 18-79)]. Pre-surgical mean radiographic anterior distal tibial angle (ADTA) was 87.8 degrees (79-100) and mean maximum dorsiflexion was -5.7 degrees (-40-7). At follow-up, mean improvement of radiologic ADTA was 18 degrees and mean clinical dorsiflexion improvement was 8.4 degrees. On the Oxford Ankle Foot Questionnaire, children reported a mean functional score of 47\% in the "Physical" domain, 71\% in "School and Play", 70\% in "Emotional", and 38\% in footwear. Parents reported mean scores of 45\% in Physical, 69\% in School and Play, 56\% in Emotional, and 28\% in footwear. Sixty percent of parents reported increased foot flexibility, $80 \%$ noted improvement in their child's walking, and 100\% would choose this surgery again.

Conclusions: Anterior distal tibial hemi-epiphysiodesis is a viable option for treatment of residual equinus in children with a clubfoot history and open growth plates. Radiographic improvement was at least $2 x$ greater than the clinically measured improvements of dorsiflexion, yet each family would undergo the surgery again as they perceived an improvement in function. 
Level of Evidence: Case series - Level IV

\section{Key Concepts:}

- Surgical management for untreated, refractory, or recurrent clubfoot continues to present a challenge to pediatric orthopaedic surgeons.

- Radiographic improvement of our patients was roughly twice that of the improvement in clinical dorsiflexion.

- Of the parents/caregivers that responded, $80 \%$ noted an improvement in their child's walking, $60 \%$ reported improved foot flexibility, and all reported they would still have their child undergo ADTH if they could go back in time.

- We feel that anterior distal tibial hemi-epiphysiodesis is an option for surgical treatment of residual equinus deformity in children with clubfoot.

\section{Introduction}

Equinus is defined as an inability to dorsiflex the ankle past neutral with the knee extended and is one of the four primary presenting features of children with clubfoot. ${ }^{1-4}$ Despite widespread use of the Ponseti method (typically including Achilles tenotomy), a certain percentage of patients with idiopathic clubfeet can develop persistent equinus as they age. This is even more common in children with nonidiopathic clubfoot such as arthrogryposis and spina bifida. Potential causes of equinus may be incomplete initial correction or recurrence, possibly as a result of nonadherence with abduction bracing treatment. In the walking child, equinus deformity can be associated with altered gait mechanics (i.e., toe walking or back knee gait) as well as foot pain, compensatory midfoot collapse, and difficulties with shoe and/or bracewear. There are several treatment considerations for equinus in older clubfoot patients. Conservative options include recasting, bracing/ orthotics, and physical therapy. Surgical treatment options include lengthening of the gastrocsoleus complex (e.g., tendo-achilles lengthening or gastrocnemius recession), posterior release (ankle and subtalar capsulotomy), and more aggressive surgical techniques involving gradual correction with ringed external fixator or supra-malleolar osteotomy. ${ }^{5-8}$

A less described technique for equinus correction is anterior distal tibia hemi-epiphysiodesis (ADTH). ${ }^{9}$ Guided growth is an accepted technique for coronal and sagittal plane deformity correction in the skeletally immature patient. Kramer and Stevens described the technique in knee flexion contractures. By tethering the anterior distal femoral physis, the remaining growth is harnessed to drive deformity correction. ${ }^{10}$ Stevens et al. hypothesized a similar technique could be utilized at anterior distal tibia to correct equinus following prior clubfoot surgery. ${ }^{11,12}$ The single published series on this technique reported limited clinical results (i.e., minimal ankle dorsiflexion improvement) despite radiographic improvement of equinus. ${ }^{9}$ The purpose of this paper is to report our experience in comparison to the only other publication. We believe there is a role for anterior distal tibial guided growth to address equinus in patients with clubfoot.

\section{Materials and Methods}

This was a single institution study; approval was obtained by the Institutional Review Board. A retrospective case review was performed investigating outcomes of pediatric clubfoot patients with equinus deformity treated via ADTH. Inclusion criteria consisted of clubfoot diagnosis, treatment by one of two fellowship trained pediatric orthopaedic surgeons between 2010-2015, and a minimum of 18 months radiographic follow-up after ADTH.

\section{Surgical Method}

The ideal patient for this technique is approximately 10 years old with equinus and radiographic features 
not amenable to soft tissue release (e.g., flat-top talus or dysmorphic changes in the talar neck) which could block dorsiflexion. ${ }^{13}$ The epiphyseal height is measured to determine appropriate implants. Given often dysmorphic anatomy, a CT scan may be helpful for preoperative planning. Commercially available modular guided growth systems typically utilize 4.0 $\mathrm{mm}$ screws, but if the patient's epiphysis is narrow, the authors have successfully used $2.7 \mathrm{~mm}$ plates cut to span the growth plate fixated with solid $2.7 \mathrm{~mm}$ screws.

The approach interval is between tibialis anterior and EHL. Care is taken to expose the area, retracting the tendinous and neurovascular structures and avoiding injury to periosteum. Under lateral fluoroscopy, a guide pin is placed in the center of the epiphysis. The plate is centered on the metaphysis in the plane of ankle motion (which may not be directly on the anterior surface of distal tibia) prior to placing solid metaphyseal screws. For those patients with smaller epiphyses, a similar strategy is employed with a 2.7 $\mathrm{mm}$ system. In this case, a $2.0 \mathrm{~mm}$ wire is temporarily driven into the appropriate position, and a solid 2.7 $\mathrm{mm}$ screw is placed into this path upon pin removal. Regardless of screw diameter, selected screw length is about one-third shorter than anterior-posterior diameter of the epiphysis.

\section{Study Design}

Medical records were examined to determine additional diagnoses, previous clubfoot treatments, clinical range of motion, operative details, additional corrective procedures following ADTH (including implant removal), and possible direct or indirect complications. Pre- and postoperative lateral weight-bearing radiographs and intraoperative fluoroscopy were reviewed to determine anterior distal tibial angle (ADTA) and screw spread angle. ADTA was measured as the angle formed between the anatomical axis of the tibia and the joint line on lateral radiograph ${ }^{9}$ (Figure 1).

We wanted to see if a change in the angulation of the screws could be related to a change in the ADTA; thus,

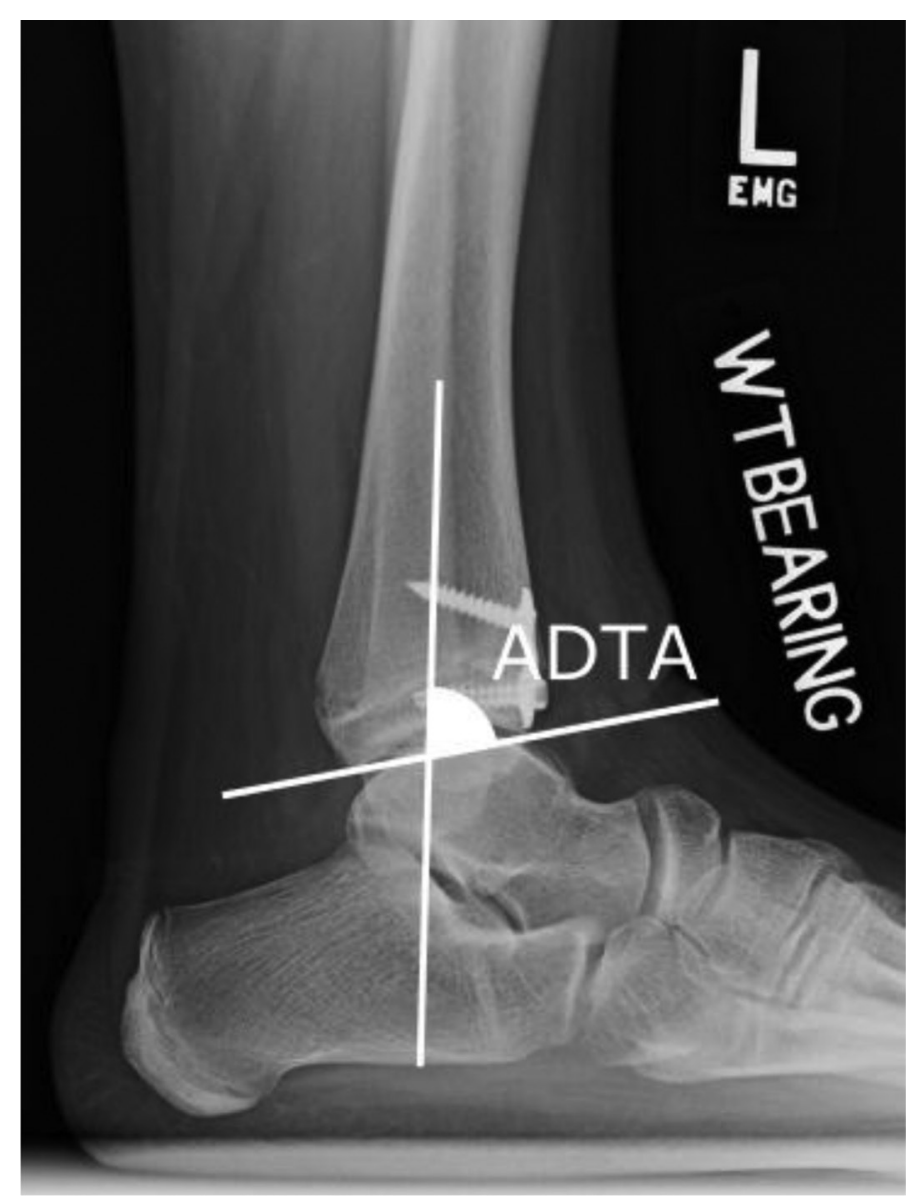

Figure 1. Anterior Distal Tibial Angle (ADTA)—Lateral weight-bearing ankle radiograph demonstrating measurement of ADTA created by the intersection of the anatomical axis of the tibia and the joint orientation line of the ankle in the sagittal plane.

screw spread was measured by divergence down the long axis of the screws (Figure 2).

All measurements were simultaneously reviewed and agreed upon by a fellowship trained pediatric orthopaedic surgeon, senior orthopaedic resident, and a medical student. Independent $t$-tests were utilized to compare differences in age at surgery, ADTA change, clinical dorsiflexion change, and radiographic follow-up length between patients with closed and open physes.

The Oxford Ankle Foot Questionnaire for Children (OxAFQ-C) and a supplemental surgery-specific questionnaire (Appendix A) were mailed to patients and their parents/caregivers to assess patient-centered 


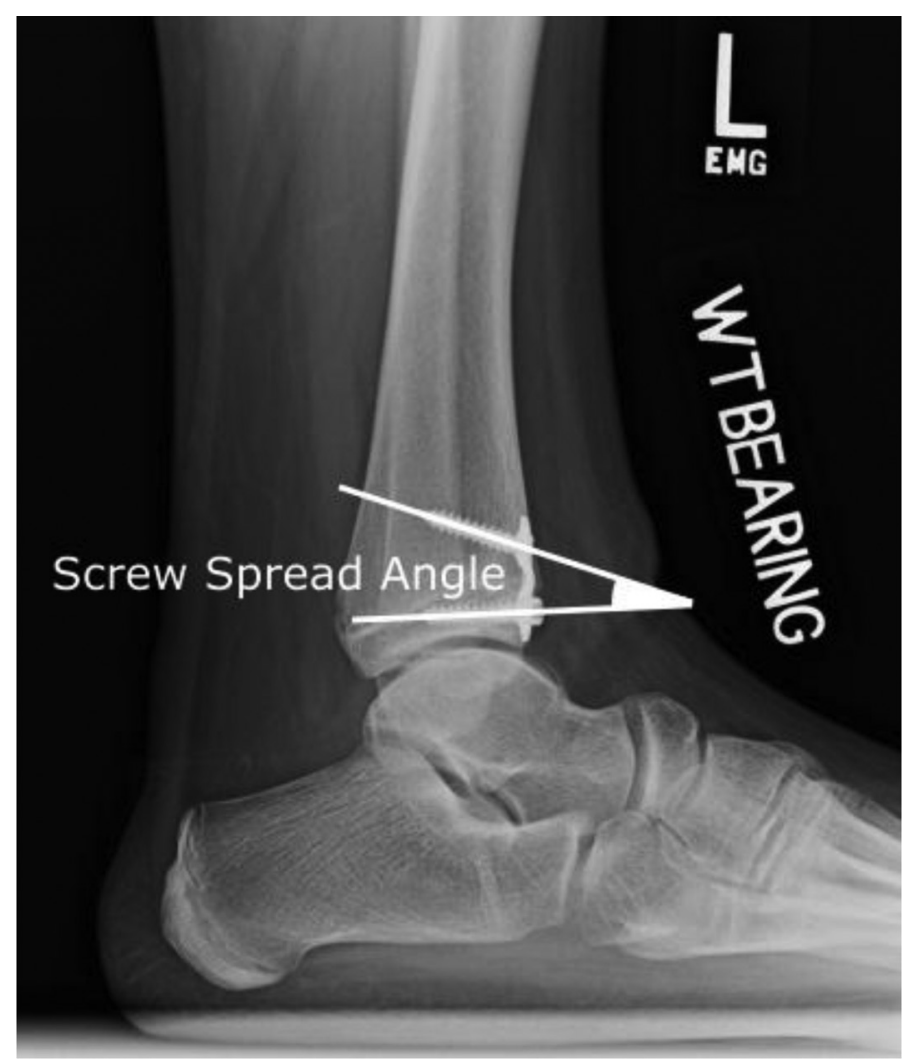

Figure 2. Screw Spread Angle-Lateral weight-bearing ankle radiograph measuring screw spread angle as determined by convergence/divergence down the long axis of fixation.

outcomes. The OxAFQ-C was utilized as a validated measure of subjective well-being in children 5-16 years of age. ${ }^{14-16}$ The OxAFQ-C is separated into three main domains: Physical Activity (6 questions), School and Play (4 questions), and Emotional (4 questions).
Additionally, one question pertaining to footwear issues is included. Responses are graded on a 5-point Likert scale (never-4, rarely-3, sometimes-2, very often-1, and always-0). Per validated guidelines, summative domain scores were converted to a percentage for ease of interpretation, with higher scores indicating better health. ${ }^{14,16}$

\section{Results}

Fourteen patients (12 males, 2 females) meeting inclusion criteria were identified (Table 1).

There was equal number (7 each) idiopathic and nonidiopathic clubfoot diagnoses, and five of the nonidiopathic patients had arthrogryposis. The remaining two cases had spastic hemiplegic cerebral palsy and craniofrontonasal syndrome (Table 2).

All patients had documented previous clubfoot treatment(s), and $93 \%$ had prior surgery. Eight patients underwent unilateral ADTH (4 Right, 4 Left) and six underwent bilateral ADTH for a total of 20 ankles. Four patients $(29 \%)$ had concurrent procedures at time of ADTH. Mean age at surgery was 10 years old (range, 4 years 2 months-12 years 8 months) and mean radiographic follow-up was 34 months (range, 1 year 6 months -6 years 7 months). At final follow-up, physeal closure was complete in five patients (36\%) accounting for seven ankles (35\%). See Table 3 for a summary of clinical characteristics.

Table 1. Patient Demographics

\begin{tabular}{|l|l|}
\hline Type & Total Number \\
\hline Patients & 14 \\
\hline Ankles & 20 \\
\hline Sex & 12 Male, 2 Female \\
\hline Laterality & 6 Bilateral, 8 Unilateral (4 Right, 4 Left) \\
\hline Mean Age at Hemi-epiphysiodesis & 10.1 years (Range 4.2-12.7 Years) \\
\hline Mean Radiographic Follow-Up & 34 Months (Range: 18-79 Months) \\
\hline Physis Closed at Follow-Up & 5 Patients, 7 Ankles \\
\hline
\end{tabular}


Table 2. Associated Diagnosis in 14 Pediatric Patients

\begin{tabular}{|l|l|}
\hline Type & Total Number \\
\hline Arthrogryposis $^{1,2}$ & 5 \\
\hline Spastic Hemiplegic Cerebral Palsy & 1 \\
\hline Craniofrontonasal Syndrome & 1 \\
\hline Hip Dysplasia ${ }^{1,2}$ & 3 \\
\hline Scoliosis $^{1}$ & 1 \\
\hline Pierre Robin Syndrome $^{2}$ & 1 \\
\hline
\end{tabular}

${ }^{1}$ Patient with concurrent Arthrogryposis, Hip Dysplasia, and Scoliosis

${ }^{2}$ Patient with concurrent Arthrogryposis, Hip Dysplasia, and Pierre Robin Syndrome

\section{Follow-up}

Preoperative mean dorsiflexion was -5.7 degrees (range, -40 to -7 ) [negative value indicating plantarflexion]. At final follow-up, mean dorsiflexion was 2.7 degrees (range, -15 to 15 ) with a mean improvement of 8.4 degrees. Preoperative mean ADTA was 87.8 degrees (range, 79-100) and mean screw divergence was 1.5 degrees (range, -8.4-7.9) determined by intraoperative fluoroscopy. At final follow-up, mean ADTA was 69.8 degrees (range, 41-90) with a mean improvement of 18 degrees. Mean

Table 3. Hemi-epiphysiodesis Patient Demographics, Treatments, and Complications

\begin{tabular}{|c|c|c|c|c|c|c|}
\hline Patient & Sex & Laterality & $\begin{array}{l}\text { Age at } \\
\text { Surgery } \\
\text { (Months) }\end{array}$ & $\begin{array}{l}\text { Concurrent } \\
\text { Procedures }\end{array}$ & Prior Treatments & $\begin{array}{c}\text { Complications and } \\
\text { Treatments Post } \\
\text { ADTH }\end{array}$ \\
\hline 1 & M & $\mathrm{L}$ & 129 & None & $\begin{array}{l}\text { Bi SC, Bi PT, Bi PMR, L Revision PMR, } \\
\text { L TAL, L Talectomy, L Navicular Excision }\end{array}$ & None \\
\hline 2 & M & $\mathrm{R}$ & 132 & None & R PMR, R TDO, R Repeat PMR & None \\
\hline 3 & M & $\mathrm{R}$ & 138 & None & Bi SC, Bi PMR, Repeat Bi PMR, R TAT & None \\
\hline 4 & M & $\mathrm{L}$ & 117 & None & L SC, L PT, L PMR, L PFR & Jones Fracture \\
\hline 5 & M & $\mathrm{L}$ & 50 & Midfoot HWR & Bi SC, Bi PT, L TDO, L CCW, L COW & None \\
\hline 6 & M & $\mathrm{Bi}$ & 116 & None & Bi SC, Bi PMR & None \\
\hline 7 & M & $\mathrm{R}$ & 139 & TAT, TAL & $\begin{array}{l}\text { Bi TAL, Bi "Clubfoot Revision" and } \\
\text { "Medial Surgery" (India) }\end{array}$ & Jones Fracture \\
\hline 8 & M & $\mathrm{R}$ & 141 & $\begin{array}{l}\text { TAT, TAL, CCW, } \\
\text { COW, PFR }\end{array}$ & Bi SC, Bi PT & None \\
\hline 9 & M & $\mathrm{Bi}$ & 96 & None & Bi SC, Bi PT, Bi TAT, Bi TDO & Repeat Bi TDO \\
\hline 10 & M & $\mathrm{Bi}$ & 152 & None & $\mathrm{Bi} \mathrm{SC}$ & None \\
\hline 11 & M & $\mathrm{Bi}$ & 102 & Bi TAL, Bi PFR & Bi SC, Bi PT & None \\
\hline 12 & $\mathrm{~F}$ & $\mathrm{Bi}$ & 113 & None & Bi SC, Bi PT, Repeat Bi PT, Bi PMR & $\begin{array}{l}\text { Bi SC, Bi PFR, R COW, } \\
\text { R CCW, R TAT }\end{array}$ \\
\hline 13 & M & $\mathrm{Bi}$ & 141 & None & $\begin{array}{l}\text { Bi SC, Bi PT, Bi TAL, Bi PR, Repeat Bi } \\
\text { PR, L AR, L ADTH }\end{array}$ & None \\
\hline 14 & $\mathrm{~F}$ & $\mathrm{~L}$ & 134 & None & $\begin{array}{l}\text { Bi SC, Bi PMR, L LR, L 1-5 Metatarsal } \\
\text { Osteotomy, L COW }\end{array}$ & $\begin{array}{c}\text { L TDO, L Tarsal } \\
\text { Dorsiflexion Closing } \\
\text { Wedge Osteotomy; } \\
\text { Calcaneus Stress } \\
\text { Fracture }\end{array}$ \\
\hline
\end{tabular}

M indicates Male, F indicates Female, L indicates Left, R indicates Right, Bi indicates Bilateral, HWR indicates Hardware Removal, SC indicates Serial Casting, TAT indicates Tibialis Anterior Transfer, TAL indicates Tendo-Achilles Lengthening, CCW indicates Cuboid Closing Wedge Osteotomy, COW indicates Cuneiform Opening Wedge Osteotomy, PFR indicated Plantar Fascia Release, PT indicates Percutaneous Tenotomy, PMR indicates Posteromedial Release, TDO indicates Tibia Derotational Osteotomy, PR indicates Posterior Release, AR indicates Anterior Release, ADTH indicates Anterior Distal Tibial Hemi-epiphysiodesis, LR indicates Lateral Release 
Table 4. Mean Radiographic and Clinical Change Following Hemi-epiphysiodesis in 14 Pediatric Patients (20 Ankles)

\begin{tabular}{|l|l|l|}
\hline Measurement & Mean \pm SD [Range], Degrees & Mean Change (Degrees) \\
\hline Radiographic ADTA Preoperative & $87.8 \pm 5.7[79-100]$ & \\
\hline Radiographic ADTA Follow-Up & $69.8 \pm 13.8[41-90]$ & $18.0(95 \%$ CI, 12.4-23.6) \\
\hline Radiographic Screw Spread at Placement & $1.5 \pm 4.2[-8.4-7.9]^{1}$ & \\
\hline Radiographic Screw Spread Follow-up & $30.7 \pm 14.3[5.6-58]$ & $29.2(95 \%$ CI, 23.2-35.2) \\
\hline Maximum Dorsiflexion Preoperative & $-5.7 \pm 10.7[-40-7]^{2}$ & \\
\hline Maximum Dorsiflexion Postoperative & $2.7 \pm 9.7[-15-15]$ & (95\% CI, 2.8-14) \\
\hline
\end{tabular}

${ }^{1}$ Screw Spread: - indicates screw tips converging, + indicates screw tips diverging

${ }^{2}$ Maximum Dorsiflexion: - indicates Plantarflexion, 0 indicates neutral positioning, + indicates Dorsiflexion

screw divergence was 30.7 degrees (range, 5.658) with a mean change of 29.2 degrees (Table 4).

When comparing patients with closed versus open physis at follow-up, there was a trend for greater clinical dorsiflexion change (15.6 degrees for closed physis and 4.5 degrees for open physis) $(\mathrm{p}=0.0597)$. ADTA improved 28.5 degrees in patients with closed physes compared to 12.3 degrees in patients with open physes $(\mathrm{p}=0.030)$ (Table 5).

Following ADTH, three patients (21\%) required additional procedures to address equinus, and two patients (14.3\%) developed a Jones Fracture. One patient also developed a calcaneus stress fracture following midfoot closing wedge osteotomy. Hardware removal was performed in 6 of the 20 ankles (30\%) due to pain at implant location (4), concern for migration into the joint (1), or surgeon discretion to prevent overcorrection (1).

Follow-up surveys were obtained from 79\% (11/14) of patients. On the Oxford Ankle Foot Questionnaire, children reported a mean functional score of $47 \%$ in the "Physical" domain, 71\% in "School and Play," 70\% in "Emotional," and 38\% in footwear. Parents/caregivers reported mean scores of 45\% in Physical, 69\% in School and Play, 56\% in Emotional, and 28\% in footwear (Table 6).

Parents/caregivers of $71 \%$ (10/14) of patients completed supplemental survey. Eighty percent (8/10) reported "toe
Table 5. Patient Comparison of Open $(n=13)$ vs. Closed $(n=7)$ Physis at Final Radiographic Follow-up

\begin{tabular}{|c|l|l|}
\hline Measurement & Mean \pm SD [Range] & p-Value \\
\hline Age at Surgery (Months) & & 0.7254 \\
\hline Open & $10.0 \pm 2.5[4.2-12.7]$ & \\
\hline Closed & $10.3 \pm 1.0[9.4-11.8]$ & \\
\hline $\begin{array}{l}\text { Radiographic ADTA } \\
\text { Change (Degrees) }\end{array}$ & $12.3 \pm 6.9[5.4-29.8]$ & $\mathbf{0 . 0 3 0 0}$ \\
\hline Open & $28.5 \pm 14.6[5.8-44.2]$ & \\
\hline Closed & & 0.0597 \\
\hline $\begin{array}{l}\text { Clinical Dorsiflexion } \\
\text { Change (Degrees) }\end{array}$ & $4.5 \pm 12.8[-22-20]$ & \\
\hline Open & $15.6 \pm 9.4[-2-30]$ & \\
\hline Closed & $49 \pm 17.2[24-79]$ & $\mathbf{0 . 0 0 0 4}$ \\
\hline $\begin{array}{l}\text { Radiographic Follow-Up } \\
\text { (Months) }\end{array}$ & $26 \pm 6.8[18-41]$ & \\
\hline Open & & \\
\hline Closed & & \\
\hline
\end{tabular}

Bold result indicates $p<0.05$

walking" preoperatively and 70\% (7/10) postoperatively. Eighty percent (8/10) noted gait improvements, $60 \%$ (6/10) reported increased foot flexibility, and 100\% $(10 / 10)$ would choose to have this procedure repeated if they could go back in time (Table 7). 
Table 6. Mean $O x A F Q-C$ Responses Compared with Those From Idiopathic Clubfoot Patients Reported by Banskota et al. ${ }^{17}$ and Duffy et al. ${ }^{18}$

\begin{tabular}{|c|c|c|c|c|c|}
\hline & Present Study & Banskota et al. & & Duffy et al. & \\
\hline & $\begin{array}{l}\text { ADTH } \\
n=9 \text { child } n=10 \text { parent }\end{array}$ & Ponseti $n=145$ child & $\begin{array}{l}\text { Ponseti } n=29 \text { child } \\
n=29 \text { parent }\end{array}$ & $\begin{array}{l}\text { Surgical } n=22 \text { child } \\
\mathrm{n}=22 \text { parent }\end{array}$ & $\begin{array}{l}\text { Control } n=25 \text { child } \\
n=26 \text { parent }\end{array}$ \\
\hline \multicolumn{6}{|l|}{ Physical } \\
\hline Child & $\begin{array}{l}46.9 \pm 25.0 \\
{[8.3-70.8]} \\
\end{array}$ & $86.0 \pm 5.1$ & $85.8 \pm 12.8$ & $77.8 \pm 20.2$ & $96.8 \pm 5.4$ \\
\hline Parent & $\begin{array}{l}45.0 \pm 25.4 \\
{[0-83.3]} \\
\end{array}$ & & $81.9 \pm 20.4$ & $74.0 \pm 20.9$ & $98.4 \pm 4.1$ \\
\hline \multicolumn{6}{|c|}{ School \& Play } \\
\hline Child & $\begin{array}{l}71.1 \pm 25.6 \\
{[43.8-100]}\end{array}$ & $98.9 \pm 4.8$ & $97.8 \pm 7.5$ & $93.5 \pm 17$ & $100 \pm 0$ \\
\hline Parent & $\begin{array}{l}68.8 \pm 26.4 \\
{[25-100]}\end{array}$ & & $95.7 \pm 11.6$ & $88.4 \pm 15.5$ & $100 \pm 0$ \\
\hline \multicolumn{6}{|c|}{ Emotional } \\
\hline Child & $\begin{array}{l}69.5 \pm 34.6 \\
{[18.8-100]} \\
\end{array}$ & $95.4 \pm 14.6$ & $92.9 \pm 9.8$ & $89.5 \pm 16.4$ & $99.8 \pm 1.3$ \\
\hline Parent & $\begin{array}{l}55.6 \pm 29.5 \\
{[12.5-100]}\end{array}$ & & $91.2 \pm 13.7$ & $75.0 \pm 21.6$ & $100 \pm 0$ \\
\hline \multicolumn{6}{|c|}{ Footwear (Single Question) } \\
\hline Child & $37.5 \pm 40.1[0-100]$ & $84.5 \pm 26.4$ & $79.3 \pm 32.8$ & $84.1 \pm 30.4$ & $99 \pm 5$ \\
\hline Parent & $27.5 \pm 34.3[0-100]$ & & $67.2 \pm 32.8$ & $54.5 \pm 35.9$ & $100 \pm 0$ \\
\hline
\end{tabular}

OxAFQ-C scores transformed to percentage scale (0-100) reported as Mean $\pm \mathrm{SD}$ [Range], \% Higher score indicates better function

Table 7. Supplemental Survey Responses $(n=10)$

\begin{tabular}{|l|l|l|}
\hline & Yes & No \\
\hline Toe walk prior to surgery? & $80 \%$ & $20 \%$ \\
\hline Did surgery help walk better? & $80 \%$ & $20 \%$ \\
\hline Did surgery increase foot flexibility? & $60 \%$ & $40 \%$ \\
\hline $\begin{array}{l}\text { If you could go back in time would you still } \\
\text { choose for your child to have surgery? }\end{array}$ & $100 \%$ & $0 \%$ \\
\hline
\end{tabular}

\section{Discussion}

Over the last 15 years of performing ADTH, the senior authors have found it is effective for improving ankle motion and function in clubfoot patients with equinus. The purpose of this study was to evaluate our intermediate term results in patients with idiopathic and nonidiopathic clubfoot who were treated for equinus with ADTH. From this study, we conclude that this technique is useful for driving both radiographic and clinical improvement. With average follow-up of 34 months, we observed improved dorsiflexion on physical examination, increased ADTA and screw spread, and parent/caregiver assessed clinical improvement. This technique was associated with approximately 10 degrees of gained ankle dorsiflexion, 20 degrees of improved anterior distal tibia angle, and 30 degrees of increased screw spread. In addition, parent/caregiver retrospectively evaluated the procedure of clinical benefit.

Our results are in contrast to the paper by Al-Aubaidi et al. who published the first case series of 25 residual clubfoot patients treated with ADTH for equinus, reporting minimal clinical benefit (i.e., ankle dorsiflexion improvement of 2 degrees) despite radiographic improvement of equinus as defined by the anterior distal tibial angle (mean shift 15 degrees). ${ }^{9}$ 
Factors that could potentially impact the difference in outcomes are the longer mean radiographic follow-up period (34 months vs. 22 months) which results in a longer period of guided growth in those remaining skeletally immature throughout and the older mean age at surgery in our patient population (10 years vs. 7 years) with older patient age corresponding with accelerated growth. It should be noted that previously published animal models of guided growth at our institution demonstrated that time is needed for modular implants to begin effecting change at the physis to correct deformity. ${ }^{19}$ It is possible that better results could have been found by Al-Aubaidi et al. with longer follow-up.

Longer follow-up is needed on this patient series; however, we have noted that patients followed to skeletal maturity obtained greater dorsiflexion and change in ADTA than those with open growth plates at follow-up, suggesting those patients may continue to improve. As patients neared/achieved skeletal maturity, it may have resulted in an increased likelihood of maintaining the correction achieved and decreasing the propensity to relapse into equinus should implants be removed. This is further supported by the significant difference in radiographic ADTA change $(p=0.030)$ and near significance of clinical dorsiflexion change $(p=0.0597)$ between patients who were skeletally mature at final follow-up compared to those that were not. Anecdotally, one patient in our study underwent unilateral ADTH at a young age, achieved successful correction, had implants removed to prevent overcorrection, and subsequently relapsed into equinus (Figure 3).

This suggests that timing is an important variable in preoperative planning to obtain a successful outcome from ADTH.

Additional surgical procedures were performed following ADTH in three patients (21\%), involving five total ankles (25\%). Procedures included tibial derotational osteotomies, plantar fascia release, midfoot osteotomies, and tibialis anterior tendon transfer. Two adolescent males participating in athletics developed a Jones fracture in the follow-up period. However, these fractures were likely due to causes (notably underlying cavus) other than ADTH based on timing and presentation. Additionally, one female sustained a calcaneus stress fracture after weight-bearing following midfoot closing wedge osteotomy. We believe this may be due to the patient weight-bearing through the heel for the first time ever as she toe-walked prior to the procedures. However, due to the above pattern, increased lateralization of the ankle forces cannot be ruled out and warrants further evaluation.

To our knowledge, only two published studies utilizing the OxAFQ-C for evaluation of clubfoot patients exist. Banskota et al. evaluated Nepalese patients with

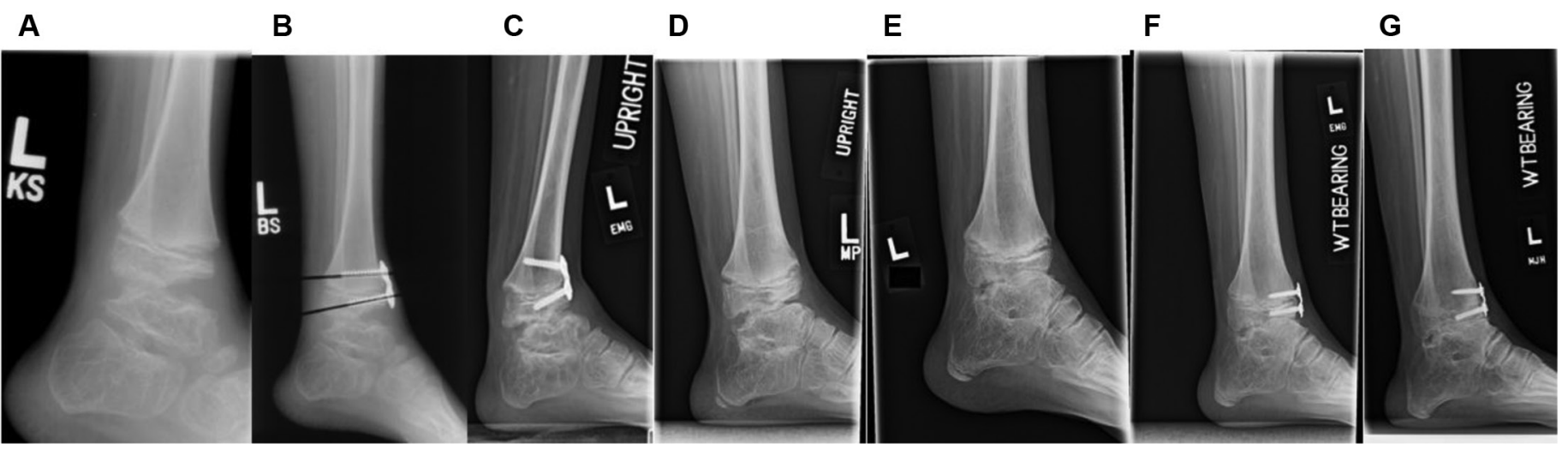

Figure 3. Series of lateral ankle radiographs in 5.5-year-old male nonidiopathic clubfoot patient with associated arthrogryposis demonstrating residual equinus deformity (A) just prior to treatment and (B) 3 months later following ADTH with modular plate. Patient achieved adequate correction (C) after 28 months and hardware was removed to prevent overcorrection. Relapse of equinus was noted 20.5 months later (D) and progressed at 4-month re-evaluation (E). It was decided to repeat ADTH (F) and increasing screw divergence is demonstrated at 2-year follow-up $(G)$. 
untreated, idiopathic clubfoot in children ages $1-5$ years old who were subsequently treated via Ponseti method with $>10$ years follow-up. ${ }^{17}$ Duffy et al. performed a comparative study of treatment via Ponseti vs. surgical management in patients with congenital clubfoot and included a control group of children without impairment. ${ }^{18}$ Both studies included only patients with idiopathic clubfoot and the number of clubfoot patients with residual equinus deformity in each study was quite small $(27 / 220,12 \%$ and $1 / 75,1.3 \%) .{ }^{17,18}$ Though we found their included populations too dissimilar to ours for detailed analysis, we found utility in general comparison of reported OxAFQ-C scores. The OxAFQ-C scores of our cohort were consistently lower than those reported across all groups in both studies. This was not entirely unexpected as half of our population had nonidiopathic clubfoot, all had residual deformity following previous treatment, and one carries a diagnosis of hemiplegic cerebral palsy which has been associated with lower OxAFQ-C domain scores. ${ }^{14}$ Nonidiopathic clubfoot, particularly when associated with arthrogryposis, is often more difficult to treat, requiring a greater number of casts to obtain initial correction, recurring at a higher rate, having lower success rates at final follow-up, and often requiring surgical management. ${ }^{20,21}$

While mean radiographic improvement of our patients was roughly twice that of the improvement in clinical dorsiflexion (18.0 vs. 8.4 degrees), and OxAFQ-C scores were consistently lower than those presented in idiopathic clubfoot patients, our supplemental surveys demonstrate that patients/caregivers consistently found benefit following ADTH. Of the parents/caregivers that responded, $80 \%$ noted an improvement in their child's walking and $60 \%$ reported improved foot flexibility. Moreover, all reported they would still have their child undergo this procedure if they could go back in time. This response is especially notable as all patients requiring additional corrective surgery and one of the two patients with a Jones fracture responded to the survey.

We acknowledge limitations to our study. Due to its retrospective design and lack of randomization, the study is inherently limited. Additionally, the small sample size hinders our statistical power. As noted above, clubfoot is a spectrum of deformity; it can be congenital, idiopathic, or acquired, and is often associated with other conditions. Another major limitation is the concurrent multiple procedures performed. This variability creates heterogeneity in the severity, concomitant diagnoses, and previous treatments of our population, confounding our conclusions. Though our response rates were quite high, (79\% OxAFQ-C, 71\% Supplemental) the presence of nonresponse bias cannot be ruled out. Due to the retrospective study design, we lack OxAFQ-C scores prior to ADTH and throughout the correction period for longitudinal comparison. In order to mitigate this, our Supplemental ADTH Questionnaire was designed to assess if patient families perceived functional improvement and, thinking retrospectively, if they found the procedure worth undergoing. As such, there is inherently recall bias as well. The lack of previously published OxAFQ-C scores in clubfoot patients treated for residual equinus and nonidiopathic clubfoot also limited our comparison.

Treatment of clubfoot in the method of Ponseti has become the standard of care. However, surgical management for untreated, refractory, or recurrent clubfoot continues to present a challenge to pediatric orthopaedic surgeons. Our cohort demonstrated increases in both radiographic and clinical dorsiflexion, and patients reported improvements in foot flexibility and walking as well as perceived benefit of the procedure. For these reasons, we feel that anterior distal tibial hemi-epiphysiodesis is an option for surgical treatment of residual equinus deformity in children with clubfoot. However, prospective longitudinal studies including serial OxAFQ-C scoring (or other outcome scoring) and clinical assessment are needed to further evaluate the utility of ADTH in achieving sustained correction of residual equinus in patients with both idiopathic and nonidiopathic clubfoot. Additionally, the long-term functional outcomes need to be monitored for overcorrection and to evaluate the impact of the joint line alteration resulting from ADTH. 


\section{Additional Links}

- POSNA Study Guide-Clubfoot: https://posna.org/ Physician-Education/Study-Guide/Clubfoot

\section{- POSNA Study Guide-Hemiepiphysiodesis} Technique: https://posna.org/Physician-Education/ Study-Guide/Hemiepiphysiodesis-Technique

\section{References}

1. Gourdine-shaw MC, Lamm BM, Herzenberg JE, et al. Equinus deformity in the pediatric patient: causes, evaluation, and management. Clin Podiatr Med Surg. 2010;27(1):25-42.

2. Shore BJ, White N, Kerr Graham H. Surgical correction of equinus deformity in children with cerebral palsy: a systematic review. J Child Orthop. 2010;4(4):277-290.

3. Roye DP, Roye BD. Idiopathic congenital talipes equinovarus. J Am Acad Orthop Surg. 2002;10(4):239-248.

4. Ponseti IV, Smoley EN. The classic: congenital club foot: the results of treatment. 1963. Clin Orthop Relat Res. 2009;467(5):1133-1145.

5. Uglow MG, Kurup HV. Residual clubfoot in children. Foot Ankle Clin. 2010;15(2):245-264.

6. Park SS, Lee HS, Han SH, et al. Gastrocsoleus fascial release for correction of equinus deformity in residual or relapsed clubfoot. Foot Ankle Int. 2012;33(12):1075-1078.

7. Tripathy SK, Saini R, Sudes P, et al. Application of the Ponseti principle for deformity correction in neglected and relapsed clubfoot using the Ilizarov fixator. J Pediatr Orthop B. 2011; 20(1):26-32.

8. Parsa A, Moghadam MH, Jamshidi MH. Relapsing and residual clubfoot deformities after the application of the Ponseti method: a contemporary review. Arch Bone Jt Surg. 2014;2(1):7-10.

9. Al-aubaidi Z, Lundgaard B, Pedersen NW. Anterior distal tibial epiphysiodesis for the treatment of recurrent equinus deformity after surgical treatment of clubfeet. J Pediatr Orthop. 2011; 31(6):716-720.

10. Kramer A, Stevens PM. Anterior femoral stapling. J Pediatr Orthop. 2001;21(6):804-807.

11. Stevens PM, Kennedy JM, Hung M. Guided growth for ankle valgus. $J$ Pediatr Orthop. 2011;31(8):878-883.

12. Stevens PM. Guided growth: 1933 to the present. Strategies Trauma Limb Reconstr. 2006;1(1):29-35.

13. Mitchell J, Bishop A, Feng Y, et al. Residual equinus after the Ponseti method: an MRI based 3-dimensional analysis. J Pediatr Orthop. 2018;38(5):e271-e277.

14. Morris C, Doll HA, Wainwright A, et al. The Oxford Ankle Foot Questionnaire for Children: scaling, reliability and validity. J Bone Joint Surg Br. 2008;90(11):1451-1456.

15. Morris C, Doll H, Wainwright A, et al. The Oxford Ankle Foot Questionnaire for Children: review of development and potential applications. Prosthet Orthot Int. 2010;34(3):238-244.

16. Morris C, Doll H, Davies N, et al. The Oxford Ankle Foot Questionnaire for Children: responsiveness and longitudinal validity. Qual Life Res. 2009;18(10):1367-1376.

17. Banskota B, Yadav P, Rajbhandari T, et al. Outcomes of the Ponseti method for untreated clubfeet in Nepalese patients seen between the ages of one and five years and followed for at least 10 years. J Bone Joint Surg Am. 2018;100(23):2004-2014.

18. Duffy CM, Salazar JJ, Humphreys L, et al. Surgical versus Ponseti approach for the management of CTEV: a comparative study. J Pediatr Orthop. 2014;33(3):326-332.

19. Noonan KJ, Halanski MA, Leiferman E, et al. Growth retardation (hemiepiphyseal stapling) and growth acceleration (periosteal resection) as a method to improve guided growth in a lamb model. J Pediatr Orthop 2016;36(4):362-369.

20. Gurnett CA, Boehm S, Connolly A, et al. Impact of congenital talipes equinovarus etiology on treatment outcomes. Dev Med Child Neurol. 2008;50(7):498-502.

21. De mulder T, Prinsen S, Van campenhout A. Treatment of non-idiopathic clubfeet with the Ponseti method; a systematic review. J Child Orthop. 2018;12(6):575-581. 


\section{Appendix A}

\section{Supplemental Anterior Distal Tibial Hemiepiphysiodesis Questionnaire}

These questions are being asked specifically about "guided growth" surgery. This is the surgery where a plate is placed on the ankle bone to help correct toe walking and/or stretch out the heel cord. An example x-ray picture of this surgery is shown below.

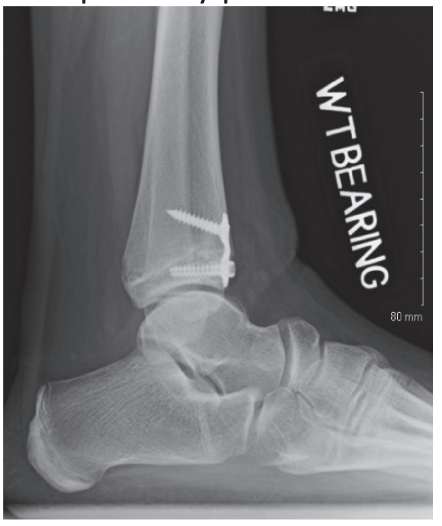
had this surgery by Dr.

on

Please feel free to include as much or as little detail as you would like or as you feel is important.

1. Before surgery, did your child toe walk?

Yes

No

2. Before surgery, what else do you remember being done for your child's foot/feet (stretching, physical therapy, bracing, orthotics, casting, other surgery(ies)?

3. Did this surgery help your child walk better?

Yes No

4. Did this surgery make your child's foot more flexible?

Yes

No

5. Did your child have any complications or unexpected consequences because of this surgery? If yes, please explain.

Yes No

6. After this surgery, what (if any) additional treatments were needed to treat your child's foot/feet (stretching, physical therapy, bracing, orthotics, casting, other surgery(ies)?

7. If you could go back in time, would you still choose to have your child have this surgery?

No 